\title{
Modelling and Implementation of Network Coding for Video
}

\author{
Can Eyupoglu \\ Istanbul Commerce University, Department of Computer Engineering, Istanbul, 34840, Turkey \\ E-mail: ceyupoglu@ticaret.edu.tr \\ Ugur Yesilyurt \\ Takasbank, Department of Application Development, Istanbul, 34381, Turkey \\ E-mail: uyesilyurt@takasbank.com.tr
}

\begin{abstract}
In this paper, we investigate Network Coding for Video (NCV) which we apply for video streaming over wireless networks. NCV provides a basis for network coding. We use NCV algorithm to increase throughput and video quality. When designing NCV algorithm, we take the deadline as well as the decodability of the video packet at the receiver. In network coding, different flows of video packets are packed into a single packet at intermediate nodes and forwarded to other nodes over wireless networks. There are many problems that occur during transmission on the wireless channel. Network coding plays an important role in dealing with these problems. We observe the benefits of network coding for throughput increase thanks to applying broadcast operations on wireless networks. The aim of this study is to implement NCV algorithm using C programming language which takes the output of the H.264 video codec generating the video packets. In our experiments, we investigated improvements in terms of video quality and throughput at different scenarios.
\end{abstract}

Index Terms-Network coding, video streaming, wireless networks.

\section{INTRODUCTION}

Wireless networks suffer from low throughput and cannot provide high quality video because of the irregular and time varying nature of wireless channel [1-4]. In video streaming over wireless, there are many researches related to cross layer design, wireless networking, video compression and streaming [1, 5-7].

Network coding is a new research field and can be used in many applications. Furthermore, it is applied to practical networking systems. In network coding, intermediate nodes may send out packets which are linear combinations of previously received information. There are two main benefits of network coding. These are throughput improvement and high robustness [8]. Moreover, network coding is utilized in wireless networks [9-12], P2P file distribution [13, 14], ad-hoc sensor networks [15], network tomography [16, 17] and network security [18-20].

In order to improve network throughput, Network
Coding for Video (NCV) has been proposed and seen as a promising method [21, 22]. The main idea of NCV algorithm is to select the best network code in order for improving video quality. Different scheduling schemes of video packets are generated by NCV algorithm ensuring an increase in throughput. Each video packet has a different contribution in a video signal. The best network code is reconstructed by taking the importance of different chosen packets and their corresponding flows [1, 23].

The rest of the paper is organized as follows. Section II discusses related work. In Section III, Network Coding for Video algorithm is explained. Section IV investigates the simulation results that show the improvements in video quality and throughput. Finally, conclusions being under study are summarized in Section V.

\section{RELATED WORK}

A wireless mesh network example is shown in Fig. 1. I is an intermediate node. $\mathrm{A}, \mathrm{B}$ and $\mathrm{C}$ are receiving or sending nodes. In video streaming over wireless mesh networks, intermediate nodes can forward packets to other intermediate nodes or clients.

In order to maximize video quality and throughput, many algorithms have been developed and they can be used at intermediate nodes. Network coding operations are performed at intermediate nodes. In addition, intermediate nodes combine packets from several incoming streams into a single outgoing packet. Then, this outgoing packet is broadcasted to the entire neighborhood. Therefore, it reaches lots of nodes concurrently. It is assumed that all nodes can overhear all transmissions in their neighborhood. Besides, they can decode network coded packets using overheard packets [1, $24,25]$.

It is observed that when video streams are transmitted, video quality must also be considered in addition to the best choice. In order for selecting the codes which contribute to the video stream quality, the importance and deadlines of video packets must be taken into consideration [1].

There are many terminologies used in network coding. These are shown in Fig. 2. 


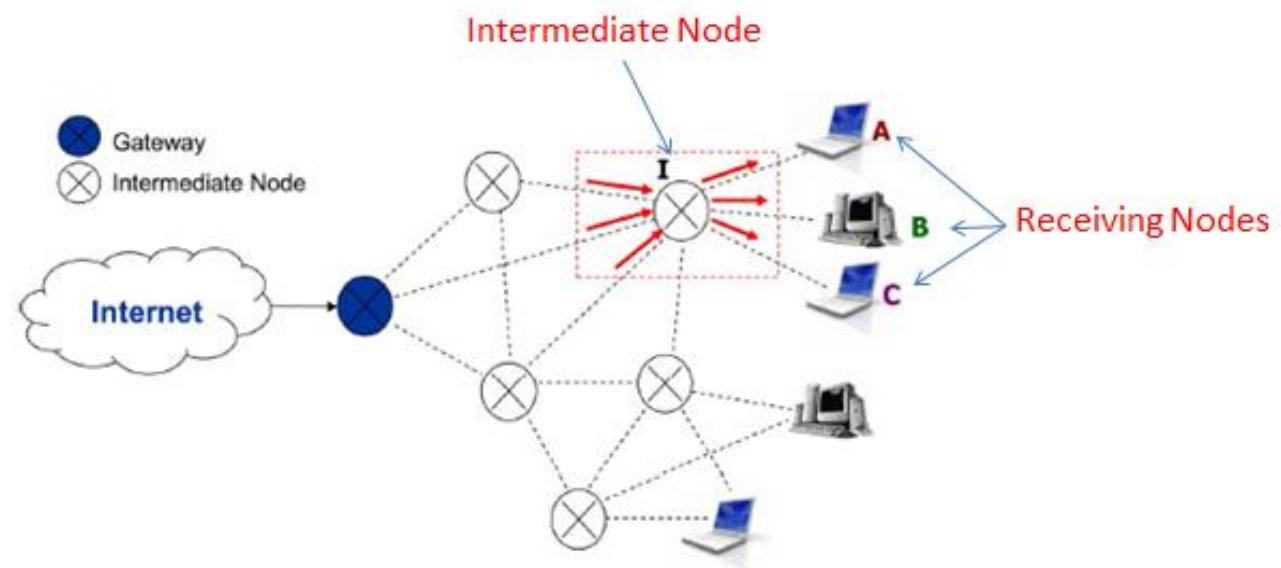

Fig.1. A Wireless Mesh Network Example.

Primary packet is the packet selected from the Tx queue before network coding. It must be included in all network codes. Furthermore, it can be thought as the main packet in a given time-slot. Side packet is the packet in the Tx queue, other than the primary, included in the network code. In network code, one primary and all side packets are XOR-ed together into a single packet. Active packet is the packet in the Tx queue that can be considered as primary. Inactive packet is the packet in the Tx queue that cannot be considered as primary. Target node is the intended recipient of the primary packet. Tx queue is the output queue of the transmitting node. Rx buffer is the receive queue of the receiving node. It stores received packets, destined to this node. Virtual buffer is also maintained at a receiving node. It stores overheard packets, destined to other nodes [1].

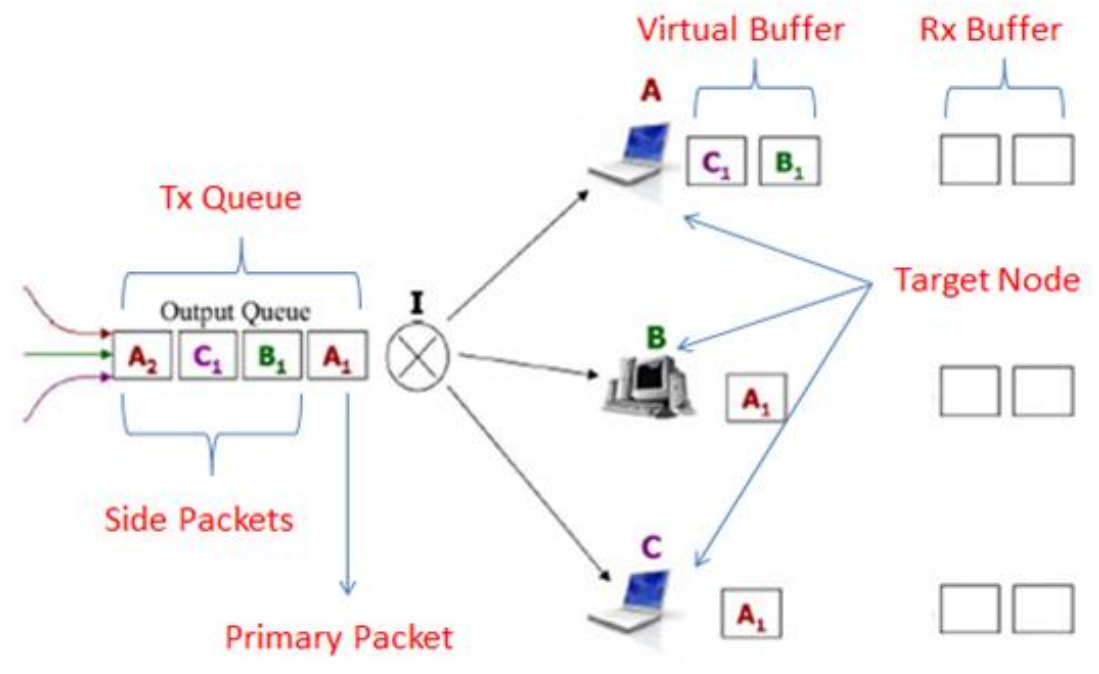

Fig.2. Network Coding Terminologies.

In code selection, it is considered that an intermediate node receives $N$ packets from different video streams. Then, it forwards these packets to $N$ nodes in its neighborhood. The intermediate node maintains a Tx queue with incoming packets. In order for transmission, a packet is selected from the Tx queue at a given time slot. The selected packet is called the primary packet. In addition, its destination is called the target node. The primary packet is considered as the main packet which is desired to transmit during a time slot. The primary packet might become the first packet or any packet in Tx queue that is marked as active depending on the network coding scheme. Besides, all packets are considered as candidate side packets. The side and primary packets are XOR-ed together into a single packet. This is called the network code. The main point is to choose the best network code in order to maximize the total video quality and throughput [1].

\section{METHOD}

\section{A. NCV Algorithm}

$\mathrm{NCV}$ algorithm is one of the methods developed for improving network throughput. Moreover, it selects the 
network codes maximizing video quality. NCV guarantees throughput increase thanks to creating various scheduling schemes of video packets. In a video signal, each video packet has a different contribution. Furthermore, taking the importance of different chosen packets and their corresponding flows, the best network code is formed.

In $\mathrm{NCV}$, the packets which come to an intermediate node are held on Tx queue. The queue has FIFO algorithm. The first packet of the queue is the primary packet and others are side packets. The primary packet and side packets are XOR-ed to create possible network codes. The target node is the intended recipient of the primary packet. If the target node can get the network code successfully, it can decode it.

Nodes have two kinds of buffers. One of them is virtual buffer which is for overheard packets. Overheard packets are related to previous transmissions. Other one is $\mathrm{Rx}$ buffer which is for received packets. The primary packet which comes to target node is stored in Rx buffer and ACK is sent to the intermediate node. The time between the beginning of transmission and getting an ACK is round trip time (RTT). In this time period, the sent packets are marked inactive in Tx queue. When ACK comes from the related node, the packets which are in network code are erased from Tx queue. If the ACK doesn't come during RTT, the video packets will be active again and the process will be repeated. The packets which are in Tx queue are held until the packets are transmitted or the deadline is missed. Nodes can encode and decode in real time [1]. NCV algorithm is shown in Fig. 3.

1: Initialization: $I_{\max }^{i}=0, c_{\max }^{i}=\emptyset$

2: Choose the first head-of-queue active packet as primary $p_{i}$.

3: Let $t\left(p_{i}\right)$ be the target node of packet $p_{i}$. Let $\left\{\nu_{1}, \ldots, \nu_{\Psi_{t}\left(p_{i}\right)}\right\}$ be the overheard packets at $t\left(p_{i}\right)$.

4: for $k=1 \ldots 2^{\psi_{t}\left(p_{i}\right)}$, do

index of primary packet $\quad k^{\text {th }}$ subset of overheard packets

5: $\quad c_{k}^{i}=\left\{p_{i}\right\} \bigcup \widehat{S_{k}^{t\left(p_{i}\right)}}$

index of subset of overheard packets

index of primary packet in the Tx queue

6: Calculate $I_{k}^{i}$ with $I_{k}^{i}=\sum_{n=1}^{N \longleftarrow} I_{k}^{i}\left(n_{\eta}\right)$ number of clients

7: $\quad$ if $I_{k}^{i}>I_{\max }^{i}$ then

8: $\quad I_{\max }^{i}=I_{k}^{i}, c_{\max }^{i}=c_{k}^{i}$

9: $\quad$ end if

10: end for

11: Choose $c_{\max }^{i}$ as the network code. XOR all packets and transmit

Fig.3. NCV Algorithm [1].

- In the first step of the algorithm, we initialize the maximum improvement to zero and the best network code to empty set. In the beginning, there is no calculated value.

- Second, we choose the first active packet in Tx queue at the intermediate node as a primary packet.

- Third, we find the target node of the chosen primary packet and create a subset of overheard packets in the target node.

- Then, $k$ is the number of possible network codes. The maximum value of $k$ is denoted by 2 over number of overheard packets.

- The chosen primary packet and the subset of overheard packets are combined and thus possible network code is formed.

- For every possible network code, the improvement at each client is calculated and summed up.

- In 7th step, we compare the new improvement value and the previous one.

- This comparison continuous until finding the maximum improvement. Then, we choose the best network code that provides the maximum improvement. 
- At the end of the algorithm, we find the best network code and then all packets in this code are XOR-ed and transmitted to clients.
The step 6.1 and 6.1.1 of NCV algorithm are shown in Fig. 4 and 5, respectively.

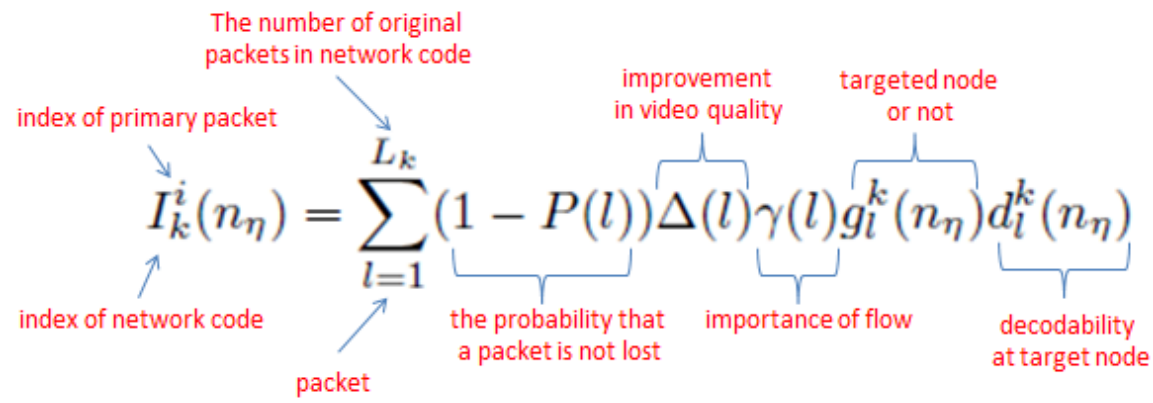

Fig.4. The Step 6.1 of NCV Algorithm.

- According to the step 6.1, first, the improvement of each code is calculated to find the best network code. To calculate the improvement of each code, the lost probability of packets, distortion value of packets, priority of the corresponding flow and indicator functions of network code are considered. Indicator functions are related to the decodability of the packet and also whether the current node is the real target node.

- If the priorities of all flows are equal, the value of priority of flow becomes 1 for all. We define indicator function $d$ if the code is decodable at the node becomes 1 or 0 otherwise. If the packet is targeted to the node, indicator function $g$ becomes 1 or 0 otherwise.

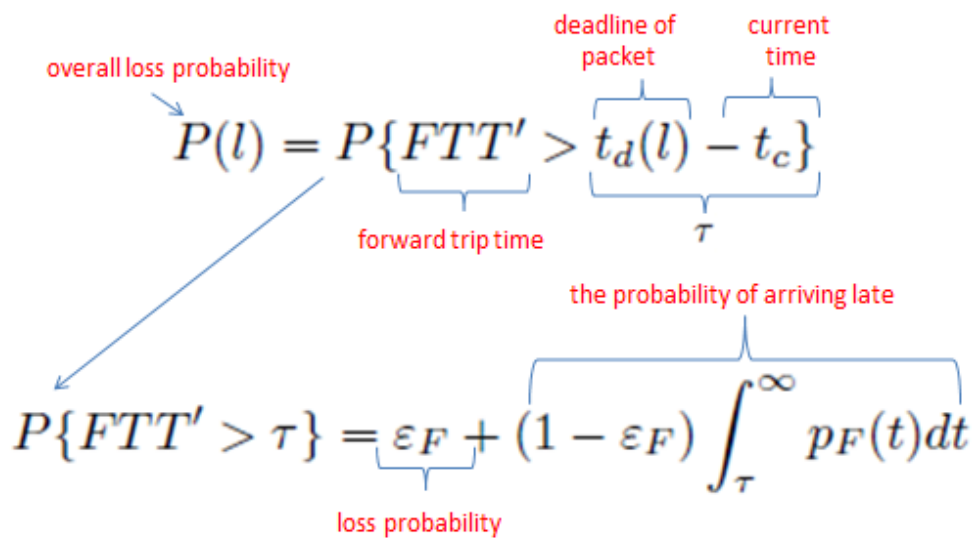

Fig.5. The step 6.1.1 of NCV Algorithm.

- According to the step 6.1.1, overall loss probability of a packet is related to the remaining time to the deadline of a packet and forward trip time (FTT) which is based on delay and loss. If FTT is greater than the remaining time, the packet will be lost.

- Loss probability is calculated with adding loss probability due to noise, fading and interference and the probability of arriving late after its deadline.

\section{B. NCV Algorithm Examples}

In the first example (Fig. 6), we try to choose the best network code for $A_{1}$ primary packet. There are four possible network codes. It takes account two side nodes $B_{1}$ and $C_{1}$ besides the primary packet $A_{1}$. If we take account $A_{2}$, the number of possible network codes will increase and will be 8 . The number of possible codes can be determined with 2 over number of side packets.
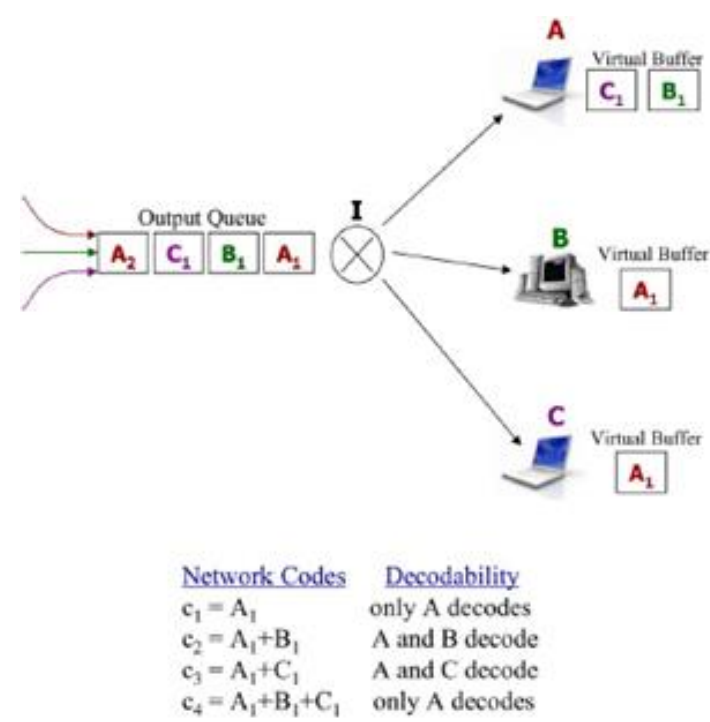

Fig.6. The First Example of NCV Algorithm. 
$\mathrm{c}_{1}$ network code contains only $\mathrm{A}_{1}$ packet and it can be decoded by only node $\mathrm{A}$ because $\mathrm{A}_{1}$ can be decoded without any help of overheard packets. Other nodes ignore $A_{1}$ because $A_{1}$ is not related to other nodes.

$c_{2}$ network code contains $A_{1}$ and $B_{1}$ packets. This code can be decoded by $\mathrm{A}$ and $\mathrm{B}$ nodes. With the help of the overheard packets in node $\mathrm{A}, \mathrm{c}_{2}$ network code can be decoded because node $A$ has overheard packet $B_{1}$. In addition, $\mathrm{c}_{2}$ network code can be decoded with the help of the overheard packet in node $\mathrm{B}$ because node $\mathrm{B}$ has overheard packet $\mathrm{A}_{1}$.

$c_{3}$ network code contains $A_{1}$ and $C_{1}$ packets. $A$ and $C$ nodes can decode this code. With the help of the overheard packets in node $\mathrm{A}, \mathrm{c}_{3}$ network code can be decoded because node $A$ has overheard packet $C_{1}$. Besides, $c_{3}$ network code can be decoded with the help of the overheard packet in node $\mathrm{C}$ because node $\mathrm{C}$ has overheard packet $A_{1}$.

$\mathrm{c}_{4}$ network code contains $\mathrm{A}_{1}, \mathrm{~B}_{1}$ and $\mathrm{C}_{1}$ packets. Only A node can decode this code. With the help of the overheard packets in node $\mathrm{A}, \mathrm{c}_{4}$ network code can be decoded because node $A$ has overheard packet $B_{1}$ and $C_{1}$. Others cannot decode it.

As a result of this scenario, $c_{2}$ and $c_{3}$ are the best possible network codes. If you ask that which code will be selected? It depends on the priority of $B_{1}$ and $C_{1}$. The code which has high priority is selected.

In the second example (Fig. 7), we try to choose the best network code for $\mathrm{B}_{1}$ primary packet. There are four possible network codes. Because it takes account two side nodes $C_{1}$ and $A_{2}$ besides the primary packet $B_{1}$.
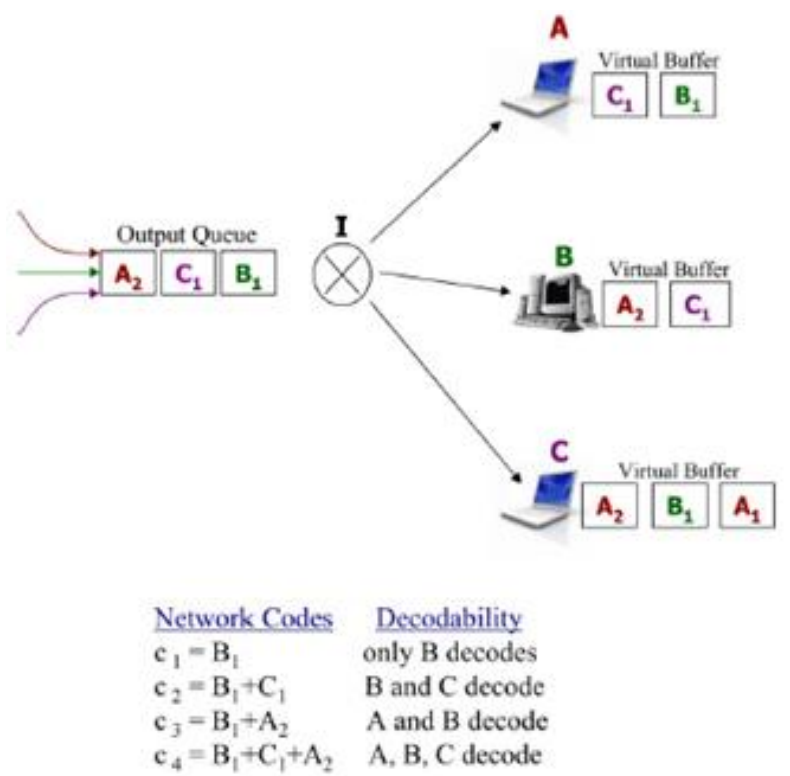

Fig.7. The Second Example of NCV Algorithm.

$\mathrm{c}_{1}$ network code contains only $\mathrm{B}_{1}$ packet and it can be decoded by only node $\mathrm{B}$, since $\mathrm{B}_{1}$ can be decoded without any help of overheard packets. Other nodes ignore $B_{1}$, because $B_{1}$ is not related to other nodes.

$c_{2}$ network code contains $B_{1}$ and $C_{1}$ packets. This code can be decoded by $\mathrm{B}$ and $\mathrm{C}$ nodes. With the help of the overheard packets in node $\mathrm{B}, \mathrm{c}_{2}$ network code can be decoded because node $\mathrm{B}$ has overheard packet $\mathrm{C}_{1}$. Furthermore, with the help of the overheard packet in node $\mathrm{C}, \mathrm{c}_{2}$ network code can be decoded because node $\mathrm{C}$ has overheard packet $\mathrm{B}_{1}$.

$\mathrm{c}_{3}$ network code contains $\mathrm{B}_{1}$ and $\mathrm{A}_{2}$ packets. $\mathrm{A}$ and $\mathrm{B}$ nodes can decode this code. With the help of the overheard packets in node $\mathrm{A}, \mathrm{c}_{3}$ network code can be decoded because node $A$ has overheard packet $B_{1}$. In addition, with the help of the overheard packet in node $B$, $\mathrm{c}_{3}$ network code can be decoded because node $\mathrm{B}$ has overheard packet $\mathrm{A}_{2}$.

$\mathrm{c}_{4}$ network code contains $\mathrm{B}_{1}, \mathrm{C}_{1}$ and $\mathrm{A}_{2}$ packets. A, B and $\mathrm{C}$ nodes can decode this code. With the help of the overheard packets in node $\mathrm{A}, \mathrm{c}_{4}$ network code can be decoded because node $A$ has overheard packet $C_{1}$ and $B_{1}$. Also, with the help of the overheard packets in node $\mathrm{B}, \mathrm{c}_{4}$ network code can be decoded because node $\mathrm{B}$ has overheard packet $A_{2}$ and $C_{1}$. Finally, with the help of the overheard packets in node $\mathrm{C}, \mathrm{c}_{4}$ network code can be decoded because node $C$ has overheard packet $A_{2}$ and $B_{1}$. As a result of this scenario, $\mathrm{c}_{4}$ is the best possible network code.

\section{NCV Algorithm Simulation}

This simulation (Fig. 8) shows how the improvement is calculated. In Tx queue, every packet has an index. In the first step, when considering the overheard packets, there are two possible network codes. These are $c_{1}=A_{1}$ and $\mathrm{c}_{2}=\mathrm{A}_{1}+\mathrm{B}_{1}$. To take improvements into account at each client, we look at the possible network codes of primary packet $A_{1}$. Besides, these improvement values are summed up for each possible network code.

In the second step (Fig. 9), after $A_{1}$ and $B_{1}$ are sent and removed from Tx queue, $C_{1}$ becomes the next primary packet which has the index number 3 . Furthermore, in this situation, there is only one possible network code which is $\mathrm{c}_{1}=\mathrm{C}_{1}$. Then, we can see the changes in $i$ and $k$ values.

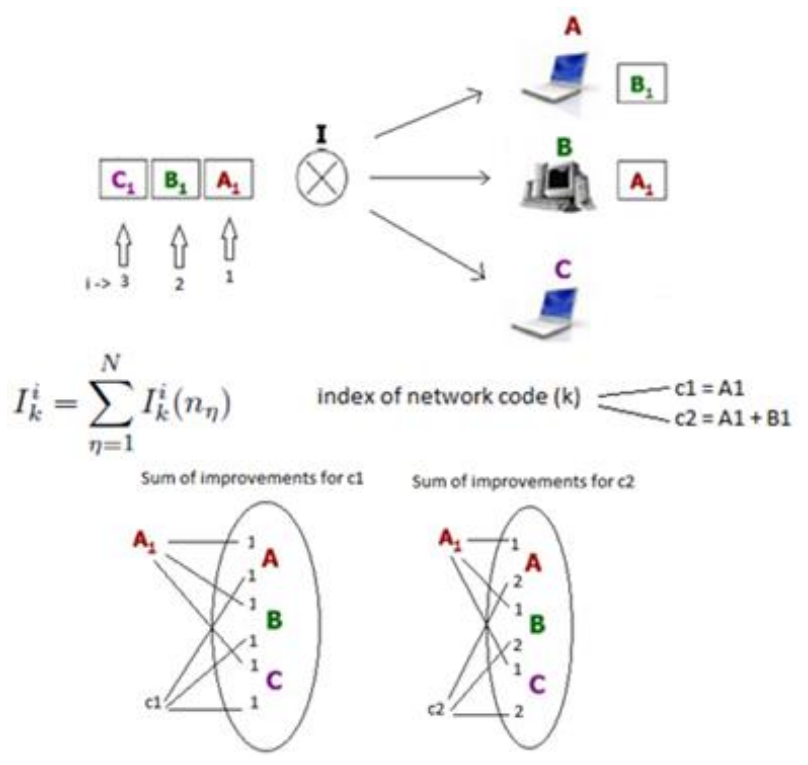

Fig.8. The First Step of NCV Algorithm Simulation. 


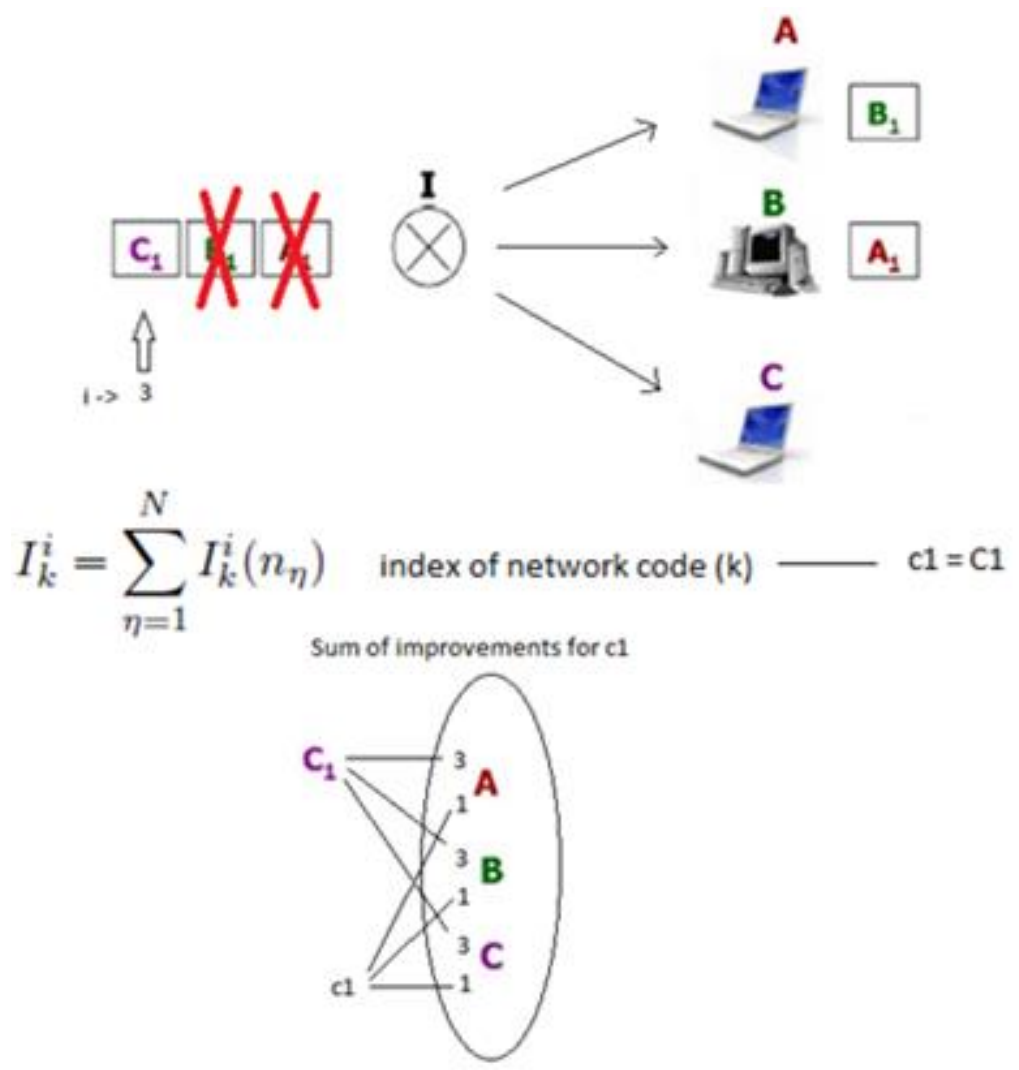

Fig.9. The Second Step of NCV Algorithm Simulation.

\section{RESULTS AND DISCUSSION}

In this study, NCV algorithm which takes the output of the H.264 video codec [26] generating the video packets is implemented in $\mathrm{C}$ programming language using Dev$\mathrm{C}++$. In addition, XEmacs text editor is used to view the contents of video frames. The main menu of developed program is shown in Fig. 10.

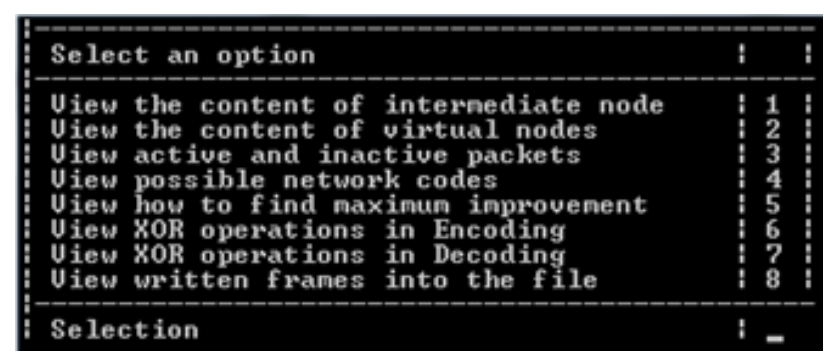

Fig.10. The Main Menu of Developed Program.

Using the main menu, desired results can be viewed by entering the number of requested option. In testing process, a football video was used and the frames were read from video packet files correctly. The system assigned names and other attributes. Besides, it converted the characters in video packet file into binary format. As an example, the attributes of a frame is shown in Fig. 11.

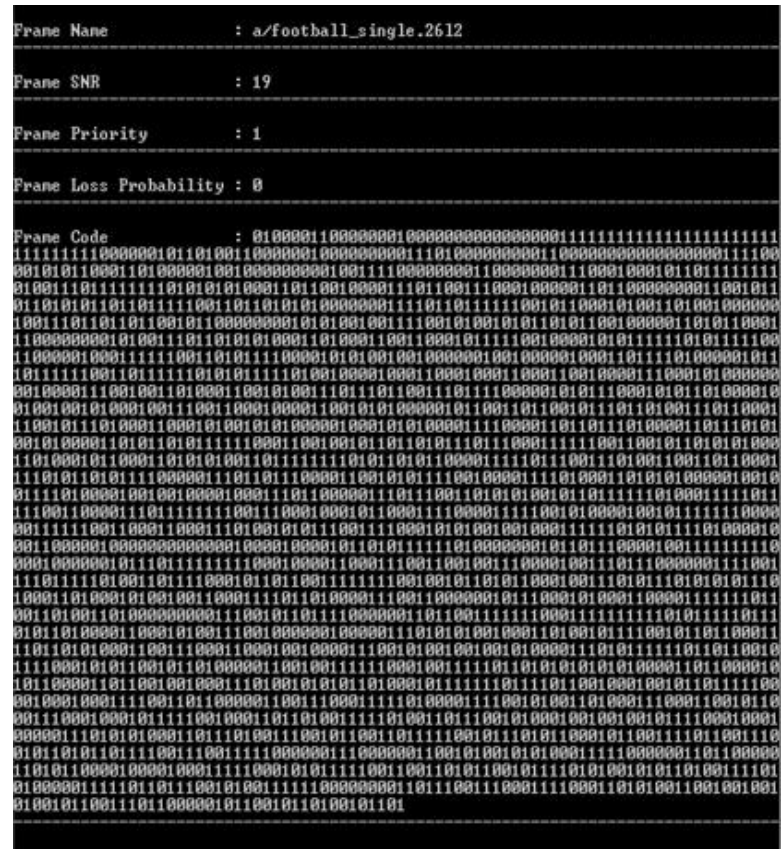

Fig.11. The Attributes of a Frame.

We confirmed that there was no missing packet after reading from the file. There were 300 packets in the intermediate node queue and 100 packets for each node. These packets are shown in Fig. 12. Then, it was seen that the active and inactive packets were listed correctly after creating each network code. These packets are shown in Fig. 13. 


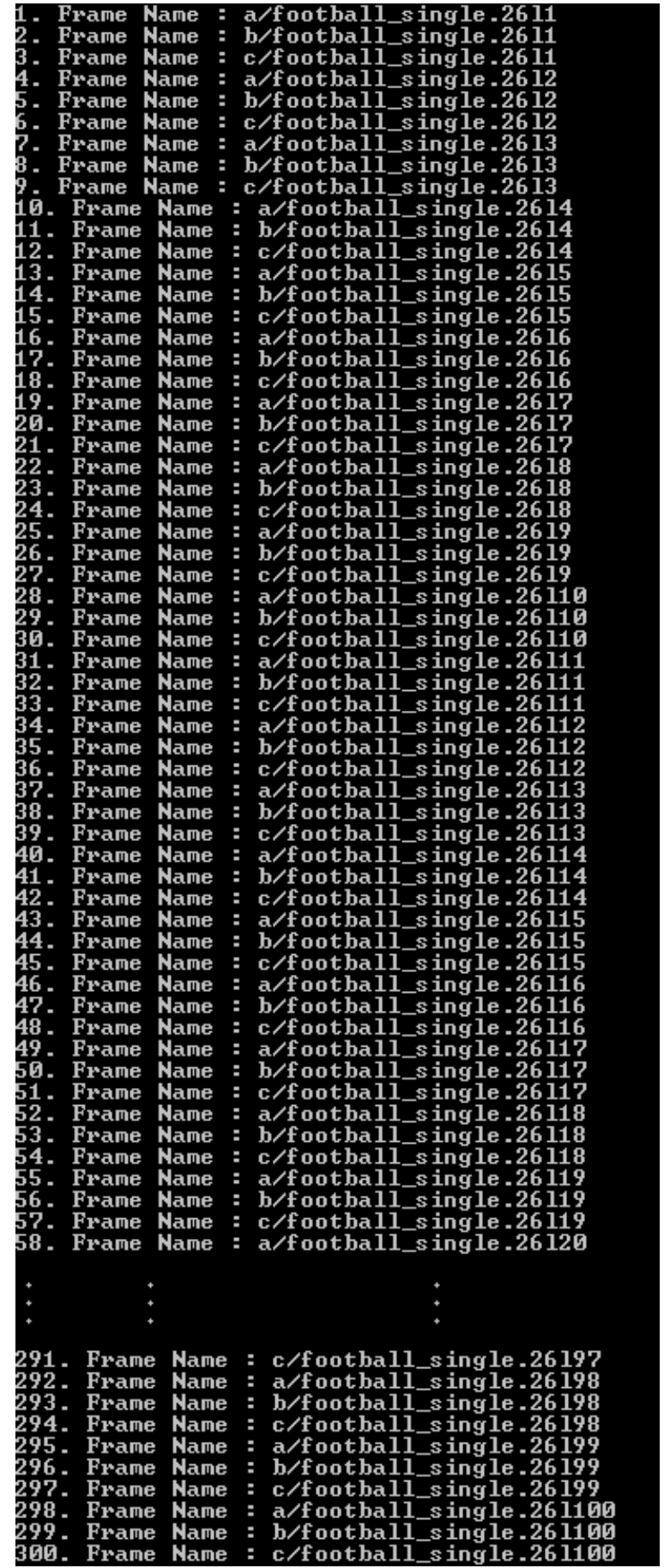

Fig.12. The Packets in the Intermediate Node Queue.

After that possible network codes were created correctly (Fig. 14). The developed program found the best network code that provides maximum improvement according to each improvement values and the same process was performed for every possible network code frame groups (Fig. 15). Then, the maximum code frames were XOR-ed correctly at encoding process and the best network code was obtained (Fig. 16). The best network code and the overheard packet code were XOR-ed accurately in order to obtain desirable frames (Fig. 17). Then, the transmission occurred and the frames were placed in the result folder after network coding (Fig. 18). Finally, the contents of frames were viewed the same as the original using XEmacs text editor (Fig. 19).
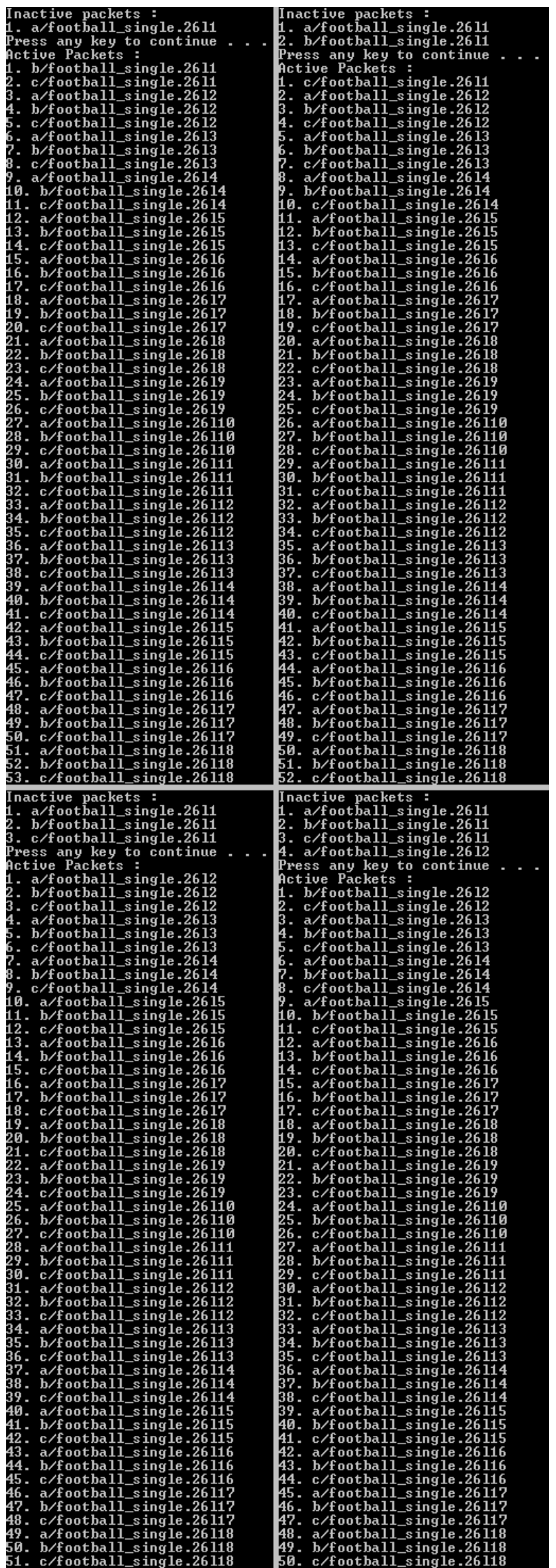

Fig.13. The Active and Inactive Packets. 


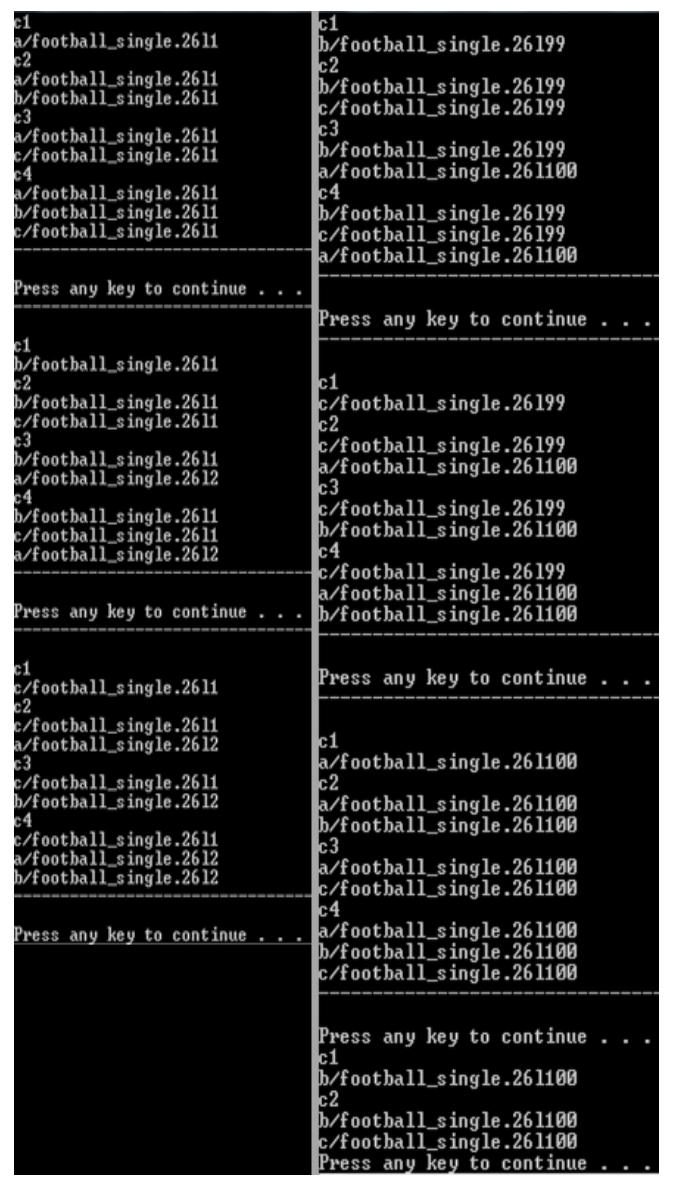

Fig.14. Possible Network Codes.

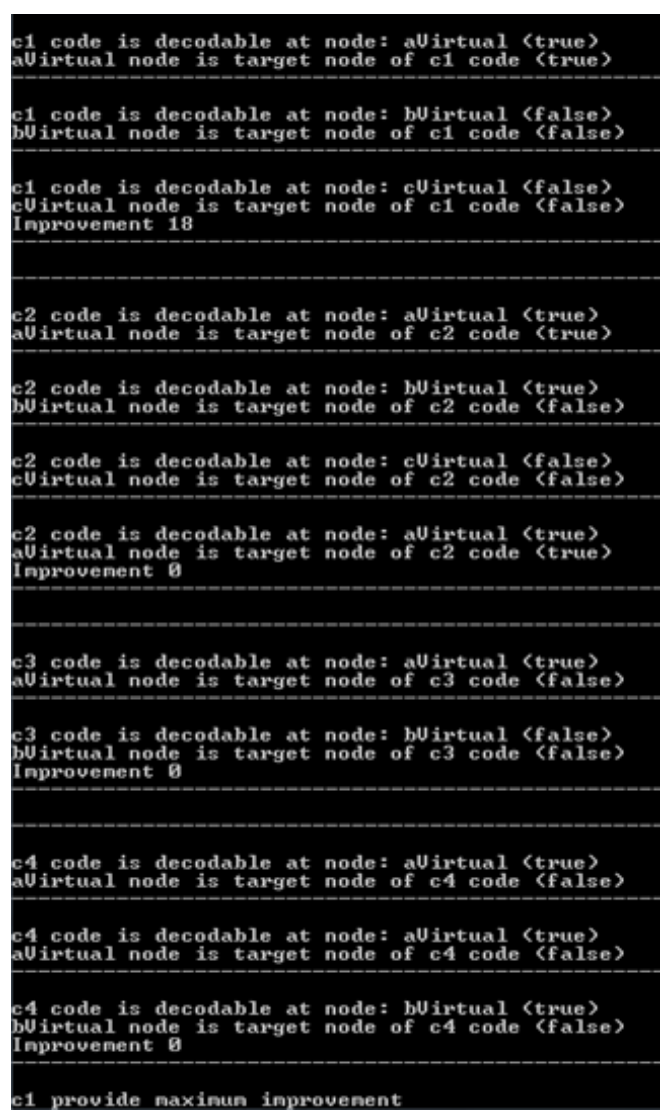

Fig.15. Finding the Best Network Code According to Each Improvement Values.

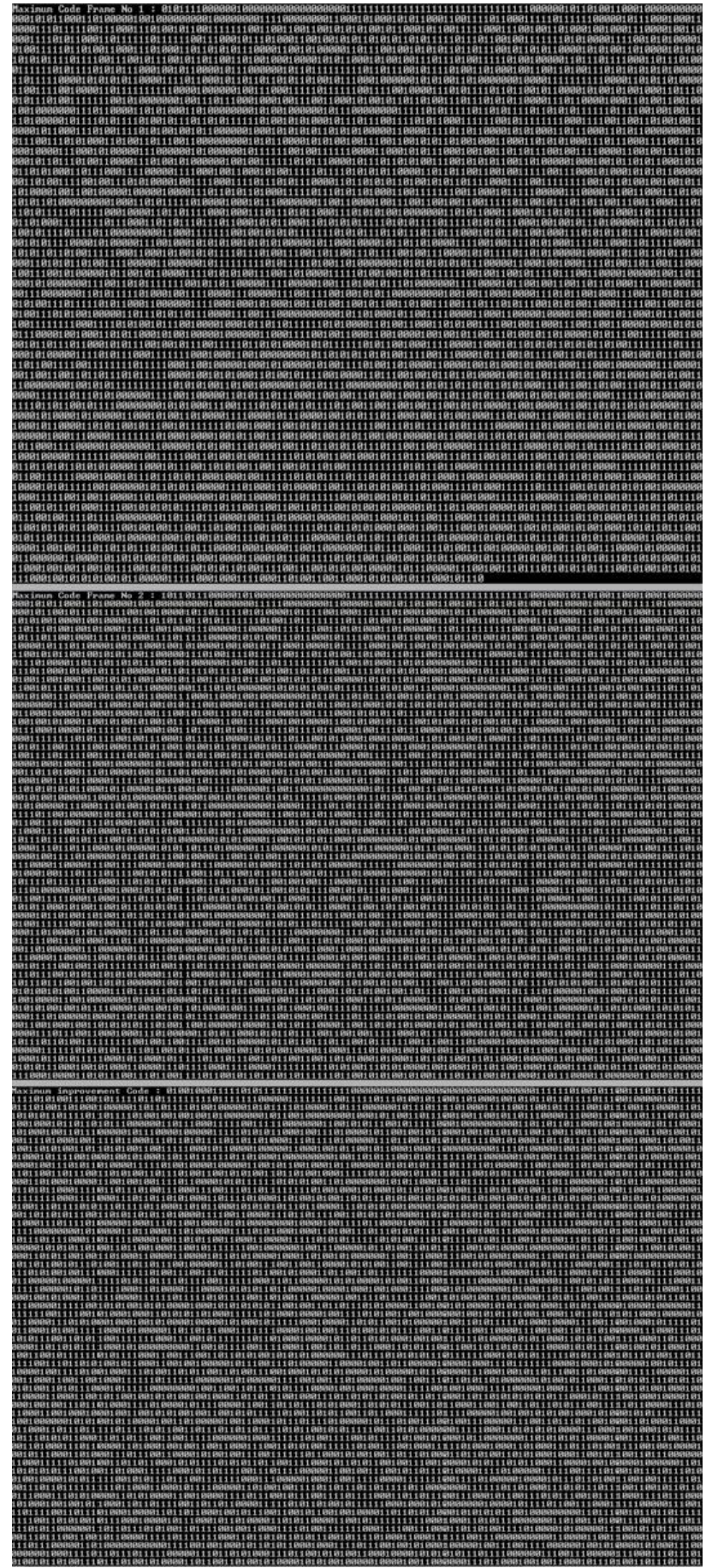

Fig.16. XOR-ing Maximum Code Frames and Obtaining the Best Network Code 

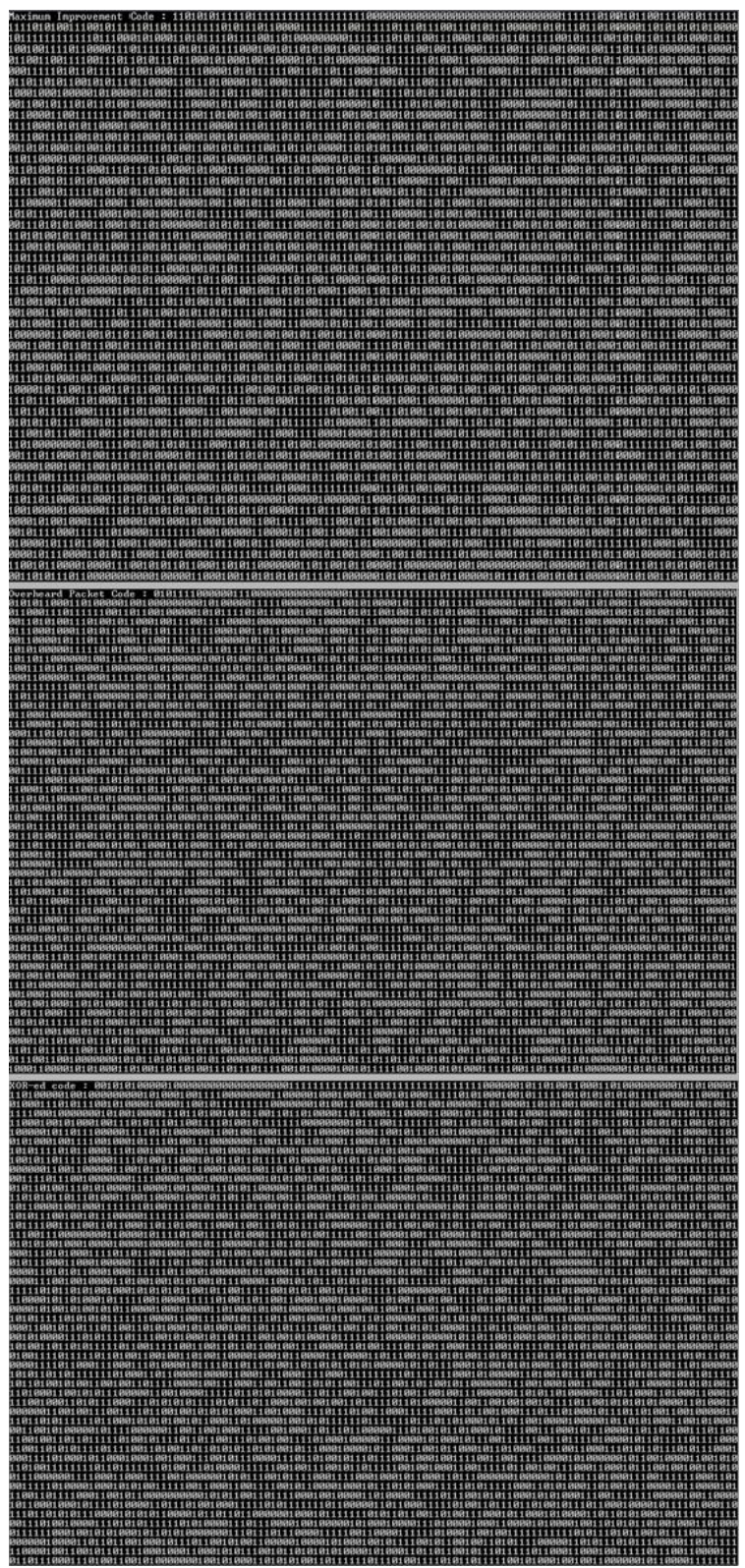

Fig.17. XOR-ing the Best Network Code and the Overheard Packet Code.

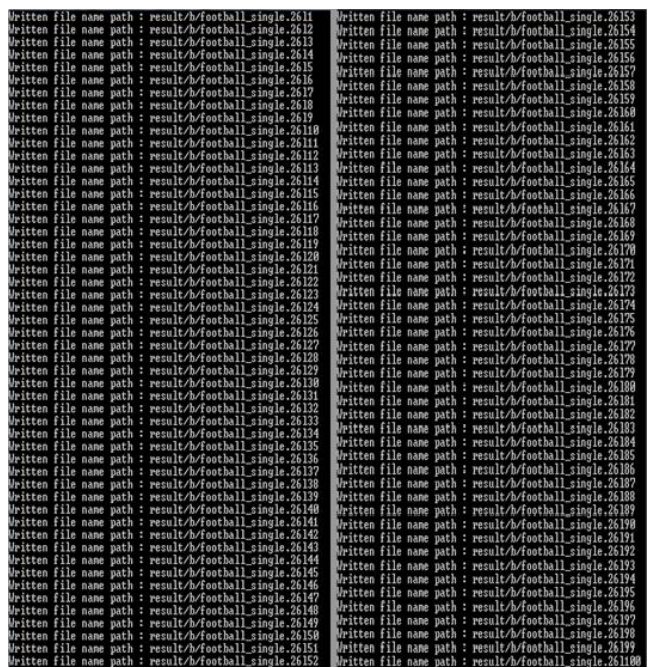

Fig. 18. Placing the Frames in Result Folder after Network Coding.

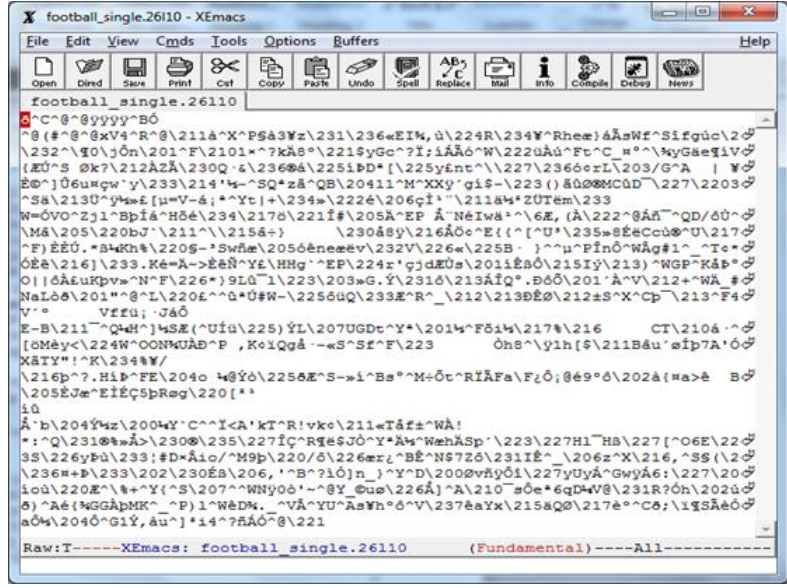

Fig.19. Viewing the Contents of Frames after Network Coding.

\section{CONCLUSION}

In this study, in order to increase throughput and video quality during video streaming over wireless networks, NCV algorithm is used. This algorithm is implemented in $\mathrm{C}$ programming language and takes the output of the H.264 video codec that generates the video packets.

The important point is to transmit flows which increase both video quality and throughput. Additionally, the deadlines of packets, the condition of the network, overheard packets on neighbor nodes and the importance of video packets are taken into account. In NCV, many flows may come to an intermediate node. In this situation, network codes are generated by looking at the buffers of clients. NCV algorithm chooses the best network code from all possible codes. The content of assistive virtual buffers of clients is utilized to get an intended primary packet from the network code. In addition to chosen primary packet, the chosen side packets must be overheard by the target node. When choosing the right network code, the most important problem is to determine whether the candidate codes can be decoded at target node or not. The network code is decoded by all neighboring nodes simultaneously. Improvement is calculated for each possible code on all receiving nodes. Finally, the improvement which has the biggest value gives the network code and this code improves video quality. According to chosen network codes, video transmission occurs. It is observed that NCV algorithm improves video quality and throughput according to the experiments performed in this study.

\section{ACKNOWLEDGMENT}

We would like to thank our project advisor Assist. Prof. Dr. Sıla Ekmekçi FLIERL for continuous assistance and contribution throughout the project process.

\section{REFERENCES}

[1] H. Seferoglu and A. Markopoulou, "Video-Aware Opportunistic Network Coding over Wireless Networks," IEEE Journal on Selected Areas in Communications, vol. 
27, no. 5, pp. 713-728, June 2009.

[2] S. Katti, H. Rahul, W. Hu, D. Katabi, M. Medard, and J. Crowcroft, "XORs in the Air: Practical Wireless Network Coding," IEEE/ACM Transactions on Networking, vol. 16, no. 3, pp. 497-510, June 2008.

[3] D. Aguayo, J. Bicket, S. Biswas, G. Judd, and R. Morris, "Link-level measurements from an $802.11 \mathrm{~b}$ mesh network," Proceedings of the 2004 Conference on Applications, Technologies, Architectures, and Protocols for Computer Communications (SIGCOMM '04), Portland, OR, pp. 121-132, Aug. 2004.

[4] H. Shiang and M. van der Schaar, "Multi-user video streaming over multi-hop wireless networks: A distributed, cross-layer approach based on priority queuing," IEEE Journal on Selected Areas in Communications, vol. 25, no. 4, pp. 770-785, May 2007.

[5] B. Girod and N. Farber, "Wireless Video," Compressed Video over Networks, A. Reibman and M.-T. Sun, Eds. New York: M. Dekker, pp. 124-133, 2000.

[6] Special issue on "Advances in Wireless Video," IEEE Wireless Communications Magazine, Aug. 2005.

[7] B. Girod, J. Chakareski, M. Kalman, Y.J. Liang, E. Setton, and R. Zhang, "Advances in Network-adaptive Video Streaming," Proceedings of IWDC 2002, Capri, Italy, Sept. 2002.

[8] C. Fragouli. J.Y. Le Boudec, and J. Widmer, "Network coding: an instant primer," ACM SIGCOMM Computer Communication Review, vol. 36, no. 1, pp. 63-68, Jan. 2006.

[9] Y. Wu, P.A. Chou, and S.-Y. Kung, "Information exchange in wireless networks with network coding and physical-layer broadcast," Technical Report MSR-TR2004-78, Microsoft Research, Aug. 2004.

[10] C. Fragouli, J. Widmer, and J.Y. Le Boudec, "A network coding approach to energy efficient broadcasting: from theory to practice," Technical Report LCA-REPORT2005-009, accepted at Infocom 2006, EPFL, July 2005.

[11] J. Widmer, C. Fragouli, and J.Y. Le Boudec, "Energy efficient broadcasting in wireless ad hoc networks," Proceedings of First Workshop on Network Coding, March 2005.

[12] J. Widmer and J.Y. Le Boudec, "Network coding for efficient communication in extreme networks," Proceedings of Workshop on delay tolerant networking and related networks (WDTN-05), Philadelphia, PA, Aug. 2005.

[13] Avalanche: File swarming with network coding. http://research.microsoft.com/pablo/avalanche.aspx.

[14] C. Gkantsidis and P. Rodriguez, "Network coding for large scale content distribution," Proceedings of IEEE Infocom, Miami, FL, Mar. 2005.

[15] D. Petrovic, K. Ramchandran, and J. Rabaey, "Overcoming untuned radios in wireless networks with network coding," Proceedings of First Workshop on Network Coding, Theory, and Applications (NetCod), Italy, Apr. 2005.

[16] C. Fragouli and A. Markopoulou, "A network coding approach to overlay network monitoring," Proceedings of Allerton Conference, Sept. 2005.

[17] T. Ho, B. Leong, Y. Chang, Y. Wen, and R. Koetter, "Network monitoring in multicast networks using network coding," Proceedings of International Symposium on Information Theory (ISIT), 2005.

[18] N. Cai and R.W. Yeung, "Secure network coding," Proceedings of International Symposium on Information Theory (ISIT), 2002.

[19] K. Bhattad and K.R. Nayayanan, "Weakly secure network coding," Proceedings of First Workshop on Network Coding, Theory, and Applications (NetCod), Apr. 2005.

[20] S-Y. R. Li, R.W. Yeung, and N. Cai, "Linear network coding," IEEE Transactions on Information Theory, vol. 49, no. 2, pp. 371-381, Feb. 2003.

[21] Q. Dong, J. Wu, W. Hu, and J. Crowcroft, "Practical Network Coding in Wireless Networks," Proceedings of MobiCom'07, Montréal, Québec, Canada, pp. 1-4, Sep. 2007.

[22] R. Ahlswede, N. Cai, S.-Y. Li, and R. W. Yeung, "Network information flow," IEEE Transactions on Information Theory, vol. 46, no. 4, July 2000.

[23] H. Seferoglu and A. Markopoulou, "Opportunistic network coding for video streaming over wireless," Proceedings of the 16thPacket Video Workshop, Lausanne, Switzerland, pp. 191-200, Nov. 2007.

[24] S. Katti, H. Rahul, W. Hu, D. Katabi, M. Medard, and J. Crowcroft, "XORs in the air: Practical wireless network coding," Proceedings of the 2006 Conference on Applications, Technologies, Architectures, and Protocols for Computer Communications (SIGCOMM'06), Pisa, Italy, pp. 243-254, Sept. 2006.

[25] S. Katti, D. Katabi, W. Hu, H. Rahul, and M. Medard, "The Importance of Being Opportunistic: Practical Network Coding For Wireless Environments," Proceedings of Allerton Conference, 2005.

[26] ITU-T Rec., H.264: Advanced video coding for generic audiovisual services, 2010.

\section{Authors' Profiles}

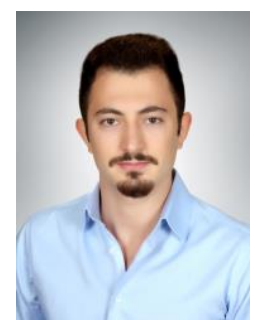

Can Eyupoglu received his B.Sc. degree (with high honor) in Computer Engineering and Minor degree in Electronics Engineering from Istanbul Kultur University, Istanbul, Turkey in 2012. He completed his M.Sc. degree in Computer Engineering from Istanbul University, Istanbul, Turkey in 2014. He is a Ph.D. candidate in Istanbul University, Department of Computer Engineering. He has been working as a Research Assistant in Istanbul Commerce University, Department of Computer Engineering since 2013. His research interests include optical networks, network security, computer arithmetic, big data and image processing.

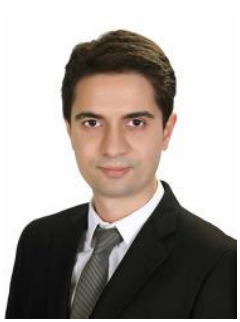

Ugur Yesilyurt received his B.Sc. degree (with honor) in Computer Engineering from Istanbul Kultur University, Istanbul, Turkey in 2012. He has been working as a Software Specialist in Takasbank, Department of Application Development since 2013. His research interests include web programming, software architecture and database management. 